\title{
Homological algebra of twisted quiver bundles
}

\author{
Peter B. Gothen ${ }^{1,2}$ and Alastair D. King ${ }^{1}$
}

30 April 2004

\begin{abstract}
Several important cases of vector bundles with extra structure (such as Higgs bundles and triples) may be regarded as examples of twisted representations of a finite quiver in the category of sheaves of modules on a variety/manifold/ringed space. We show that the category of such representations is an abelian category with enough injectives by constructing an explicit injective resolution. Using this explicit resolution, we find a long exact sequence that computes the Ext groups in this new category in terms of the Ext groups in the old category. The quiver formulation is directly reflected in the form of the long exact sequence. We also show that under suitable circumstances, the Ext groups are isomorphic to certain hypercohomology groups.
\end{abstract}

\section{Introduction}

A quiver $Q$ is a directed graph specified by a set of vertices $Q_{0}$, a set of arrows $Q_{1}$ and head and tail maps

$$
h, t: Q_{1} \longrightarrow Q_{0}
$$

Throughout the paper we shall assume that $Q$ is finite, i.e. the sets $Q_{0}$ and $Q_{1}$ are finite.

\footnotetext{
${ }^{1}$ Members of VBAC (Vector Bundles on Algebraic Curves), which is partially supported by EAGER (EC FP5 Contract no. HPRN-CT-2000-00099) and by EDGE (EC FP5 Contract no. HPRN-CT-2000-00101).

${ }^{2}$ Partially supported by the Fundação para a Ciência e a Tecnologia (Portugal) through the Centro de Matemática da Universidade do Porto and through grant no. $\mathrm{SFRH} / \mathrm{BPD} / 1606 / 2000$.
} 
Let $\mathcal{A}$ be a category. A representation of a quiver $Q$ in $\mathcal{A}$ is a correspondence which associates an object $V_{i}$ of $\mathcal{A}$ to each vertex $i \in Q_{0}$ and a morphism

$$
\phi_{a}: V_{t a} \rightarrow V_{h a}
$$

to each arrow $a \in Q_{1}$. There is an obvious notion of a morphism $f:(V, \phi) \rightarrow$ $(W, \psi)$ between representations of $Q$ : it consists of a family of morphisms $f_{i}: V_{i} \rightarrow W_{i}$ such that the diagrams

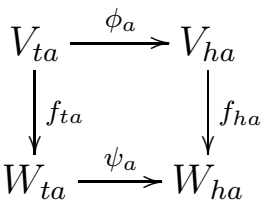

commute for every $a \in Q_{1}$. In this way the representations of $Q$ form a category; note that this is an abelian category whenever $\mathcal{A}$ is.

A fundamental case is when $\mathcal{A}$ is the category of vector spaces over a field k. We shall refer to a representation of $Q$ in this category as a $Q$-vector space or simply as a representation of $Q$. There is a related algebraic notion: given a quiver $Q$ one can construct its so-called path algebra (see Section 22) and the category of left modules over the path algebra is equivalent to the category of representations of $Q$. This has been a very fruitful idea in the theory of representations of algebras (see, e.g., Benson [5]).

Another case of great interest is when $\mathcal{A}$ is the category of algebraic vector bundles over an an algebraic variety $X$, or the category of holomorphic vector bundles over a complex manifold $X$. A representation of a quiver $Q$ in this category is called a quiver bundle or, for short, a Q-bundle. This notion unifies many of the vector bundles with extra structure which have been studied previously; we mention some examples. If $Q$ is the quiver

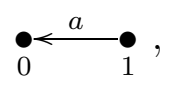

then a $Q$-bundle is a holomorphic triple as studied by García-Prada [14] and Bradlow and García-Prada [9. In the special case $V_{1}=\mathcal{O}_{X}$ this becomes equivalent to the notion of Bradlow pair [8]. More generally, consider the quiver

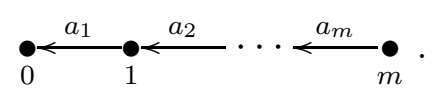

In this case a $Q$-bundle is called a holomorphic chain, these where studied by Álvarez-Cónsul and García-Prada [1. There is a generalization of the notion of $Q$-bundle to that of twisted $Q$-bundle, where the morphisms $\phi_{a}$ are twisted by a vector bundle $M_{a}$ (see Section 3 for precise definitions). An important 
example of this is that of Higgs bundles on a curve $X$, introduced by Hitchin [18]: these are pairs $(E, \phi)$, where $E$ is a vector bundle and $\phi$ is a morphism $\phi: E \longrightarrow E \otimes K_{X}$, where $K_{X}$ is the canonical bundle of $X$. Thus a Higgs bundle is a twisted $Q$-bundle where $Q$ is given by

$$
\text { Oִ } a
$$

In various applications it has been important to study infinitesimal deformations and extensions of $Q$-bundles and this has been done on a case by case basis. For Bradlow pairs, see Thaddeus [22] and for Higgs bundles see Nitsure [20], Markman [19], Bottacin [7] and Hausel and Thaddeus [17. The study of extensions of triples is important for the study of representations of the fundamental group of a surface in the group $\mathrm{U}(p, q)$ [10, 11].

The goal of this paper is to give a unified treatment of the homological algebra of twisted quiver bundles, in order to study their extensions and deformations. The natural setting is to work in an abelian category with enough injectives, but the category of $Q$-bundles is not abelian, because the category of vector bundles over $X$ is itself not abelian. We therefore work with representations of $Q$ in the category of sheaves of $\mathcal{O}_{X}$-modules on a ringed space $\left(X, \mathcal{O}_{X}\right)$, which is an abelian category and has enough injectives. In this general setting, our results apply, for example, both when $X$ is an algebraic variety with the Zariski topology and $\mathcal{O}_{X}$ is the sheaf of algebraic functions, and also when $X$ is a complex manifold with the Hausdorff topology and $\mathcal{O}_{X}$ is the sheaf of holomorphic functions. We shall call a representation of a quiver $Q$ in this category a $Q$-sheaf of $\mathcal{O}_{X}$-modules (or simply a $Q$-sheaf, for short). One sees easily that the category of $Q$ sheaves is abelian. In analogy to the case of $Q$-vector spaces, the category of $Q$-sheaves of $\mathcal{O}_{X}$-modules is equivalent to the category of sheaves of modules for a certain sheaf of algebras on $X$ (see Section 3). This more algebraic point of view is important to our approach.

As in the vector bundle situation, the notion of $Q$-sheaf generalizes to the notion of twisted $Q$-sheaf; the twisting data consists of a locally free sheaf $M_{a}$ of $\mathcal{O}_{X}$-modules for each arrow $a \in Q_{1}$ (again we refer to Section 3 for precise definitions).

Our main results are as follows. We construct an explicit injective resolution of a twisted $Q$-sheaf (Proposition 3.5); in particular it follows that the abelian category of twisted $Q$-sheaves has enough injectives. This is done by combining the standard injective resolution in the category of twisted $Q$ vector spaces with injective resolutions in the category of sheaves. From the explicit form of the injective resolution of a twisted $Q$-sheaf we then deduce a long exact sequence (Theorem 4.1) which relates the Ext groups in the 
category of twisted $Q$-sheaves to the Ext groups of the sheaves which make up the twisted $Q$-sheaves. This should be a very useful tool in practical calculations and generalizes earlier results for special quivers; it was probably first observed by Nitsure [20, in the case of Higgs bundles. Next we prove that if $V$ and $W$ are twisted $Q$-sheaves such that $V$ is locally free, then the group $\operatorname{Ext}^{i}(V, W)$ is isomorphic to a certain $i$ th hypercohomology group (Theorem 5.1). This should be useful for representing extension classes using Cech or Dolbeault cohomology, and also connects our results to the special cases mentioned above, which are generally stated using hypercohomology.

To finish this introduction, we mention some related work. On the subject of twisted $Q$-bundles, Álvarez-Cónsul and García-Prada [2, 3] have recently proved a Hitchin-Kobayashi correspondence between stable twisted $Q$-bundles and solutions to certain gauge theory equations for special metrics on the underlying smooth bundles. (In fact they consider the even more general notion of quivers with relations). This generalizes earlier results in the special cases mentioned earlier.

We also should mention the paper [6] by Biswas and Ramanan. They study the infinitesimal deformation space of principal Higgs bundles on a curve using hypercohomology. Even though this is a somewhat different situation from ours, there are certain similarities: for example they show that there is a long exact sequence relating the infinitesimal deformation space to certain sheaf cohomology groups of vector bundles associated to the underlying principal bundle.

Acknowledgements. The first author thanks The Institute for Mathematical Sciences, University of Aarhus for hospitality while part of this work was carried out.

\section{The path algebra}

In this section we recall a few relevant facts about representations of quivers and the corresponding notion of modules over the path algebra. For more details see, e.g., [4], 5], [12 or [13], but note that we use a slight modification of the standard definition of path algebra.

Let $Q$ be a quiver. A non-trivial path in $Q$ is a sequence $p=a_{m} \cdots a_{0}$ of arrows $a_{i}$, such that $h a_{i-1}=t a_{i}$, for each $i$.

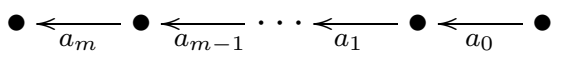

We write $t p=t a_{0}$ and $h p=h a_{m}$. In addition, for each vertex $i \in Q_{0}$, there 
is a trivial path $\langle i\rangle$, which begins and ends at $i$. The length of a non-trivial path is the number of arrows in the sequence; a trivial path has length zero.

Now, for a commutative ring $R$, suppose that we have an $R$-module $M_{a}$ for each $a \in Q_{1}$. For each non-trivial path $p=a_{m} \cdots a_{0}$, we set

$$
M_{p}=M_{a_{m}} \otimes_{R} \cdots \otimes_{R} M_{a_{0}} .
$$

For each trivial path $\langle i\rangle$, we set $M_{\langle i\rangle}=R$ and write $e_{i} \in M_{\langle i\rangle}$ for the element thereby identified with $1 \in R$.

Definition 2.1. Let $Q$ be a quiver and let $M=\left\{M_{a}\right\}_{a \in Q_{1}}$ be a collection of $R$-modules. The $M$-twisted path algebra over $R$ is

$$
A=\bigoplus_{p} M_{p}
$$

where the sum is over all paths in $Q$ and the $R$-linear multiplication is defined by

$$
x_{p} x_{q}=x_{p} \otimes x_{q} \text { if } t p=h q, \text { and } 0 \text { otherwise, }
$$

for $x_{p} \in M_{p}$ and $x_{q} \in M_{q}$, while

$$
\begin{aligned}
& x_{p} e_{i}=x_{p} \text { if } i=t p, \text { and } 0 \text { otherwise, } \\
& e_{i} x_{p}=x_{p} \text { if } i=h p, \text { and } 0 \text { otherwise, } \\
& e_{i} e_{j}=e_{i} \text { if } i=j, \text { and } 0 \text { otherwise. }
\end{aligned}
$$

Remark 2.2. (1) The $R$-algebra $A$ is graded, by the subspaces corresponding to paths of length $n$, that is

$$
A_{n}=\bigoplus_{|p|=n} M_{p}
$$

Indeed, $A$ may also be defined as the tensor algebra, over the ring $A_{0}=$ $\bigoplus_{i \in Q_{0}} R$, of the $A_{0}$-bimodule $A_{1}=\bigoplus_{a \in Q_{1}} M_{a}$.

(2) When each $M_{a}=R$ (or is free with one generator), then we get the usual path algebra of the quiver.

(3) Suppose that $a$ and $b$ are arrows with the same head and tail. If we replace them by one arrow $c$ and let $M_{c}=M_{a} \oplus M_{b}$, then we clearly get the same algebra.

The notion of a representation of $Q$ in the category of $R$-modules defined in the Introduction can be generalized to take into account twisting by the modules $M_{a}$ as follows: an $M$-twisted representation of $Q$ consists of $R$ modules $V_{i}$ for $i \in Q_{0}$ and $R$-module maps

$$
\phi_{a}: M_{a} \otimes_{R} V_{t a} \longrightarrow V_{h a}
$$


for $a \in Q_{1}$. A morphism between twisted representations $(V, \phi)$ and $(W, \psi)$ is a collection of $R$-module maps $f_{i}: V_{i} \longrightarrow W_{i}$ such that the diagrams

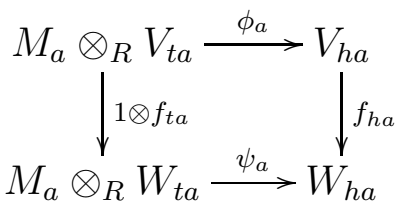

commute for all $a \in Q_{1}$. It follows immediately that the forgetful functors $(V, \phi) \mapsto V_{i}$ are exact, for each $i \in Q_{0}$.

It is a straightforward exercise to show directly that the category of $M$ twisted representations of $Q$ is an abelian category, using the fact that the category of $R$-modules is abelian and the functor $M_{a} \otimes_{R}-$ is right exact. However, this is also evident from the following.

Proposition 2.3. The category of twisted representations of $Q$ is equivalent to the category of left A-modules.

Proof. Let $(V, \phi)$ be a twisted representation of $Q$. We can then define a left $A$-module $V$ as follows: as an $R$-module it is

$$
V=\bigoplus_{i \in Q_{0}} V_{i}
$$

and the $A$-module structure is extended linearly from

$$
e_{i} v=\left\{\begin{array}{ll}
v, & v \in V_{i}, \\
0, & v \in V_{j}
\end{array} \text { for } j \neq i,\right.
$$

for $i \in Q_{0}$ and

$$
x_{a} v= \begin{cases}\phi_{a}\left(x_{a} \otimes v\right), & v \in V_{t a}, \\ 0, & v \in V_{j} \quad \text { for } j \neq t a,\end{cases}
$$

for $x_{a} \in M_{a}$ and $a \in Q_{1}$. This construction can be inverted as follows: given a left $A$-module $V$ we set $V_{i}=e_{i} V$ for $i \in Q_{0}$ and define the map $\phi_{a}: M_{a} \otimes_{R} V_{t a} \longrightarrow V_{h a}$ by $x_{a} \otimes v \longmapsto x_{a} v$. One quickly checks that morphisms of representations of $Q$ correspond to $A$-module homomorphisms (see [4] or [5] for details).

We will exploit this result and henceforth describe a twisted representation $(V, \phi)$ of $Q$ as just an $A$-module $V$. Note in particular that we have a canonical identification $V_{i}=e_{i} V$.

The following $A$-module resolution is of fundamental importance to us. 
Proposition 2.4. Let $V$ be a module over the twisted path algebra A. There is an exact sequence

$$
\begin{aligned}
& 0 \longrightarrow V \stackrel{\epsilon}{\longrightarrow} \bigoplus_{i \in Q_{0}} \operatorname{Hom}_{R}\left(e_{i} A, V_{i}\right) \\
& \stackrel{d}{\longrightarrow} \bigoplus_{a \in Q_{1}} \operatorname{Hom}_{R}\left(M_{a} \otimes_{R} e_{t a} A, V_{h a}\right) \longrightarrow 0,
\end{aligned}
$$

where

$$
\epsilon(v)(x)=x v
$$

and the map $d$ is given by

$$
d:\left(\alpha_{i}\right)_{i \in Q_{0}} \mapsto\left(\alpha_{h a} \circ \mu_{a}-\phi_{a} \circ\left(1 \otimes \alpha_{t a}\right)\right)_{a \in Q_{1}}
$$

where $\mu_{a}: M_{a} \otimes e_{t a} A \rightarrow e_{h a} A$ is the multiplication map.

Proof. Note that there is a natural inclusion

$$
\operatorname{Hom}_{A}(A, V) \hookrightarrow \bigoplus_{i \in Q_{0}} \operatorname{Hom}_{R}\left(e_{i} A, V_{i}\right)
$$

and that, under this inclusion, $\epsilon$ is just the standard isomorphism $V \stackrel{\cong}{\rightarrow}$ $\operatorname{Hom}_{A}(A, V)$. In particular, $\epsilon$ is injective.

Next we show that $\operatorname{im}(\epsilon)=\operatorname{ker}(d)$. Let

$$
\alpha=\left(\alpha_{i}\right)_{i \in Q_{0}} \in \bigoplus_{i \in Q_{0}} \operatorname{Hom}_{R}\left(e_{i} A, V_{i}\right)
$$

be such that $d \alpha=0$. The map $d$ can be written as $d=\left(d_{a}\right)_{a \in Q_{1}}$ with

$$
d_{a}(\alpha)\left(x_{a} \otimes x\right)=\alpha_{h a}\left(x_{a} x\right)-x_{a} \alpha_{t a}(x),
$$

and thus $d \alpha=0$ means that for any $x \in V_{t a}$ and $x_{a} \in M_{a}$ we have

$$
\alpha_{h a}\left(x_{a} x\right)=x_{a} \alpha_{t a}(x) .
$$

Hence, we see that $d \alpha=0$ if and only if $\alpha(y x)=y \alpha(x)$ for any $y \in A$; in other words if and only if $\alpha$ is $A$-linear. Therefore $\operatorname{ker}(d)=\operatorname{Hom}_{A}(A, V)$, showing that $\operatorname{im}(\epsilon)=\operatorname{ker}(d)$.

Finally, we must show that $d$ is surjective. Let

$$
\beta=\left(\beta_{a}\right)_{a \in Q_{1}} \in \bigoplus_{a \in Q_{1}} \operatorname{Hom}_{R}\left(M_{a} \otimes_{R} e_{t a} A, V_{h a}\right),
$$


then it suffices to find $\alpha$ such that $d_{a}(\alpha)=\beta_{a}$ for each $a \in Q_{1}$. Note that the grading of $A$ gives a natural grading $e_{i} A=\bigoplus_{l \geqslant 0} e_{i} A_{l}$, and that we have

$$
e_{i} A_{l}=\bigoplus_{\{a: h a=i\}} M_{a} \otimes_{R} e_{t a} A_{l-1} .
$$

We define $\alpha=\left(\alpha_{i}\right)_{i \in Q_{0}}$ by induction on $l$ as follows:

- On $e_{i} A_{0}$, we set $\alpha_{i}=0$ for all $i \in Q_{0}$.

- Assume that $\alpha$ is defined on $e_{i} A_{l-1}$ for all $i$, then we define $\alpha_{i}$ on $e_{i} A_{l}$ by setting

$$
\alpha_{i}\left(x_{a} \otimes x\right)=x_{a} \alpha_{t a}(x)+\beta_{a}\left(x_{a} \otimes x\right)
$$

for $x_{a} \otimes x \in M_{a} \otimes_{R} e_{t a} A_{l-1}$.

It is then easy to verify that $d_{a}(\alpha)=\beta_{a}$.

Let $A$ be any $R$-algebra, let $V$ be a left $A$-module, let $W$ be a right $A$-module and let $L$ be an $R$-module. There is the standard adjunction

$$
\operatorname{Hom}_{A}\left(V, \operatorname{Hom}_{R}(W, L)\right) \cong \operatorname{Hom}_{R}\left(W \otimes_{A} V, L\right) .
$$

The isomorphism relates $f$ on the left and $g$ on the right when

$$
f(v)(w)=g(w \otimes v)
$$

for all $v \in V$ and $w \in W$. This adjunction has several important consequences, and we will use similar results later in the paper. Firstly, since

$$
V_{i} \cong e_{i} A \otimes_{A} V,
$$

it follows that for any left $A$-module $V$ and for any $R$-modules $N$ and $L$ we have the adjunction formula

$$
\operatorname{Hom}_{A}\left(V, \operatorname{Hom}_{R}\left(N \otimes_{R} e_{i} A, L\right)\right) \cong \operatorname{Hom}_{R}\left(N \otimes_{R} V_{i}, L\right),
$$

and from this we deduce the following Proposition.

Proposition 2.5. Let $N$ be a free $R$ module and $L$ be an injective $R$ module. Then the left A-module

$$
\operatorname{Hom}_{R}\left(N \otimes_{R} e_{i} A, L\right)
$$

is injective for each $i \in Q_{0}$. 
Proof. We need to see that the functor

$$
\operatorname{Hom}_{A}\left(-, \operatorname{Hom}_{R}\left(N \otimes_{R} e_{i} A, L\right)\right)
$$

is exact, which it is, because, by (2.3), it is the composite of three exact functors: the forgetful functor $V \mapsto V_{i}$ is always exact, $N \otimes_{R}$ - is exact when $N$ is free, and $\operatorname{Hom}_{R}(-, L)$ is exact when $L$ is injective.

As a special case, suppose that $R=\mathbf{k}$ is a field. Then every vector space is both free and injective and the sequence (2.1) is the 'standard' injective resolution of $V$.

Example 2.6. Consider the quiver (1.1). In this case the path algebra $A$ can be identified with the polynomial algebra $\mathbf{k}[x]$. Let $V$ be a module over $\mathbf{k}[x]$, then the injective resolution (2.1) takes the form

$$
0 \longrightarrow V \stackrel{\epsilon}{\longrightarrow} \operatorname{Hom}_{\mathbf{k}}(\mathbf{k}[x], V) \stackrel{d}{\longrightarrow} \operatorname{Hom}_{\mathbf{k}}(\mathbf{k}[x], V) \longrightarrow 0
$$

where $\epsilon(v)(p)=p v$ and $d(\alpha)(p)=\alpha(x p)-x \alpha(p)$.

\section{Quiver sheaves}

Let $Q$ be a quiver and let $\left(X, \mathcal{O}_{X}\right)$ be a ringed space. In this section, we consider twisted representations of $Q$ in the category of sheaves of $\mathcal{O}_{X}$-modules on $X$. For basic definitions about ringed spaces and sheaves of $\mathcal{O}_{X}$-modules, refer to Hartshorne [16], Ch. II, Sec. 1,2,5. Note also that the category of sheaves of $\mathcal{O}_{X}$-modules on $X$ has enough injectives ([16] Prop. III.2.2).

Suppose we are given a collection $\left\{M_{a}\right\}_{a \in Q_{1}}$ of locally free sheaves of $\mathcal{O}_{X^{-}}$ modules. An $M$-twisted $Q$-sheaf of $\mathcal{O}_{X}$-modules (or a twisted $Q$-sheaf on $X$, for short) consists of $\mathcal{O}_{X}$-modules $V_{i}$ for $i \in Q_{0}$ and morphisms

$$
\phi_{a}: M_{a} \otimes_{\mathcal{O}_{X}} V_{t a} \longrightarrow V_{h a}
$$

for $a \in Q_{1}$. A morphism between twisted $Q$-sheaves $(V, \phi)$ and $(W, \psi)$ on $X$ is a collection of $\mathcal{O}_{X}$-module maps $f_{i}: V_{i} \longrightarrow W_{i}$ such that the diagrams

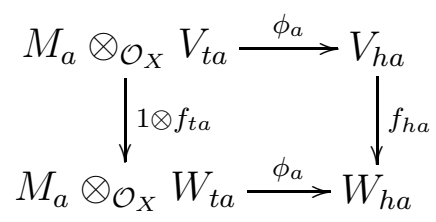

commute for all $a \in Q_{1}$. As in Section 2, the forgetful functors $(V, \phi) \mapsto V_{i}$ are exact, for each $i \in Q_{0}$, and the category of $M$-twisted $Q$-sheaf of $\mathcal{O}_{X^{-}}$ modules is abelian. 
Next we describe the corresponding algebraic notion, analogous to the definition of the twisted path algebra in Section 2. For each non-trivial path $p=a_{m} \cdots a_{0}$ in $Q$ we set

$$
M_{p}=M_{a_{m}} \otimes_{\mathcal{O}_{X}} \cdots \otimes_{\mathcal{O}_{X}} M_{a_{0}}
$$

and we set $M_{\langle i\rangle}=\mathcal{O}_{X}$. We define the $M$-twisted path algebra on $X$ to be the sheaf of $\mathcal{O}_{X}$-algebras $B=\bigoplus_{p} M_{p}$ where the sum is over all paths in $Q$ and the multiplication is the obvious one (cf. Definition 2.1).

In a similar way to Proposition 2.3 , the category of twisted $Q$-sheaves is equivalent to the category of sheaves of $B$-modules on $X$ (a detailed proof can be found in [3]). Thus we shall interchangeably use the expressions "twisted $Q$-sheaf" and "sheaf of $B$-modules", or, for short, " $B$-module".

Note that, for the elementary facts above, we do not use the assumption that the twisting sheaves $M_{a}$ are locally free, i.e. that the functor $M_{a} \otimes_{\mathcal{O}_{X}}$ is exact. However, this assumption is used in realising the main objective of this section, that is, to show that the category of twisted $Q$-sheaves has enough injectives.

Specifically, we shall construct an injective resolution of a twisted $Q$ sheaf by combining the resolution (2.1) in the $A$-module direction with an injective resolution in the $\mathcal{O}_{X}$-module direction. The first step is to generalize Proposition 2.4 to $B$-modules.

Proposition 3.1. Let $V$ be a B-module. There is an exact sequence of sheaves

$$
\begin{aligned}
0 \longrightarrow V \stackrel{\epsilon}{\longrightarrow} \bigoplus_{i \in Q_{0}} \mathcal{H o m}_{\mathcal{O}_{X}}\left(e_{i} B, V_{i}\right) \\
\stackrel{d}{\longrightarrow} \bigoplus_{a \in Q_{1}} \mathcal{H o m}_{\mathcal{O}_{X}}\left(M_{a} \otimes_{\mathcal{O}_{X}} e_{t a} B, V_{h a}\right) \longrightarrow 0
\end{aligned}
$$

where the map $\epsilon$ is defined by

$$
\epsilon(v)(x)=x v
$$

for local sections $v$ and $x$ and the map $d$ is defined on a local section $\alpha=$ $\left(\alpha_{i}\right)_{i \in Q_{0}}$ by

$$
d:\left(\alpha_{i}\right)_{i \in Q_{0}} \mapsto\left(\alpha_{h a} \circ \mu_{a}-\phi_{a} \circ\left(1 \otimes \alpha_{t a}\right)\right)_{a \in Q_{1}}
$$

where $\mu_{a}: M_{a} \otimes e_{t a} B \rightarrow e_{h a} B$ is the multiplication map. 
Proof. It is sufficient to show exactness on stalks. Let $\mathcal{O}_{x}$ be the local ring at $x$, and let $M_{a, x}, B_{x}$ and $V_{x}$ be the stalks at $x$ of $M_{a}, B$ and $V$, respectively. Recall from Serre [21, no 10] that the stalk of the tensor product of sheaves is the tensor product of the stalks over the local ring. Thus we need to show that the sequence

$$
\begin{aligned}
0 \longrightarrow V_{x} \stackrel{\epsilon}{\longrightarrow} \bigoplus_{i \in Q_{0}} \operatorname{Hom}_{\mathcal{O}_{x}}\left(e_{i} B_{x}, V_{i, x}\right) \\
\stackrel{d}{\longrightarrow} \bigoplus_{a \in Q_{1}} \operatorname{Hom}_{\mathcal{O}_{x}}\left(M_{a, x} \otimes_{\mathcal{O}_{x}} e_{t a} B_{x}, V_{x, h a}\right) \longrightarrow 0
\end{aligned}
$$

is exact for every $x \in X$. But, again using the fact on tensor products mentioned above, we see that $B_{x}$ is the $M_{a, x}$-twisted path algebra over $\mathcal{O}_{x}$. Hence exactness is immediate from Proposition 2.4.

Another important ingredient in our construction is the following analogue for sheaves of the adjunction formula (2.2).

Proposition 3.2. Let $B$ be a sheaf of $\mathcal{O}_{X}$-algebras, let $V$ a sheaf of left $B$ modules, let $W$ be a sheaf of right $B$-modules and let $L$ be an $\mathcal{O}_{X}$-module. Then there is a natural isomorphism

$$
\mathcal{H o m}_{B}\left(V, \mathcal{H o m}_{\mathcal{O}_{X}}(W, L)\right) \cong \mathcal{H o m}_{\mathcal{O}_{X}}\left(W \otimes_{B} V, L\right)
$$

Proof. This follows from (2.2) by an argument analogous to the one used in the proof of Proposition 3.1 above.

Corollary 3.3. Let $V$ be a $B$-module and $N$ and $L$ be $a \mathcal{O}_{X}$-modules. Then there is a natural isomorphism

$$
\mathcal{H o m}_{\mathcal{O}_{X}}\left(N \otimes_{\mathcal{O}_{X}} V_{i}, L\right) \cong \mathcal{H o m}_{B}\left(V, \mathcal{H o m}{ }_{\mathcal{O}_{X}}\left(N \otimes_{\mathcal{O}_{X}} e_{i} B, L\right)\right)
$$

Proof. This follows from Proposition 3.2 because $V_{i} \cong e_{i} B \otimes_{B} V$.

The following is analogous to Proposition 2.5.

Proposition 3.4. Let $L$ be an injective $\mathcal{O}_{X}$-module and let $N$ be a locally free $\mathcal{O}_{X}$-module. Then

$$
\mathcal{H o m}_{\mathcal{O}_{X}}\left(N \otimes_{\mathcal{O}_{X}} e_{i} B, L\right)
$$

is an injective $B$-module. 
Proof. We have to show that

$$
\operatorname{Hom}_{B}\left(-, \mathcal{H o m}_{\mathcal{O}_{X}}\left(N \otimes_{\mathcal{O}_{X}} e_{i} B, L\right)\right)
$$

is an exact functor. By Corollary 3.3 this functor is the composite of the three functors, $V \longmapsto V_{i}, N \otimes_{\mathcal{O}_{X}}-$ and $\operatorname{Hom}_{\mathcal{O}_{X}}(-, L)$. Just as in the proof of Proposition 2.5 the first one is always exact the second one is exact because $N$ is locally free and the third one is exact because $L$ is injective.

Now, if $V$ is any $B$-module, then for each $\mathcal{O}_{X}$-module $V_{i}$, we can choose an injective resolution

$$
V_{i} \stackrel{\epsilon}{\longrightarrow} V_{i}^{0} \stackrel{\delta}{\longrightarrow} V_{i}^{1} \stackrel{\delta}{\longrightarrow} \cdots
$$

Because $M_{a} \otimes_{\mathcal{O}_{X}}$ - is an exact functor, the usual argument, using injectivity of the $V_{h a}^{n}$, shows that the maps

$$
\phi_{a}: M_{a} \otimes_{\mathcal{O}_{X}} V_{t a} \longrightarrow V_{h a}
$$

lift to chain maps

$$
\phi_{a}^{\bullet}: M_{a} \otimes_{\mathcal{O}_{X}} V_{t a}^{\bullet} \longrightarrow V_{h a}^{\bullet}
$$

for each $a \in Q_{1}$. Thus the $V^{n}=\bigoplus_{i} V_{i}^{n}$ become $B$-modules and the maps $\delta: V^{n} \rightarrow V^{n+1}$ become $B$-module homomorphisms.

We construct the desired injective resolution of $V$ from the following double complex, which we denote by $C(V)^{\bullet \bullet}$.

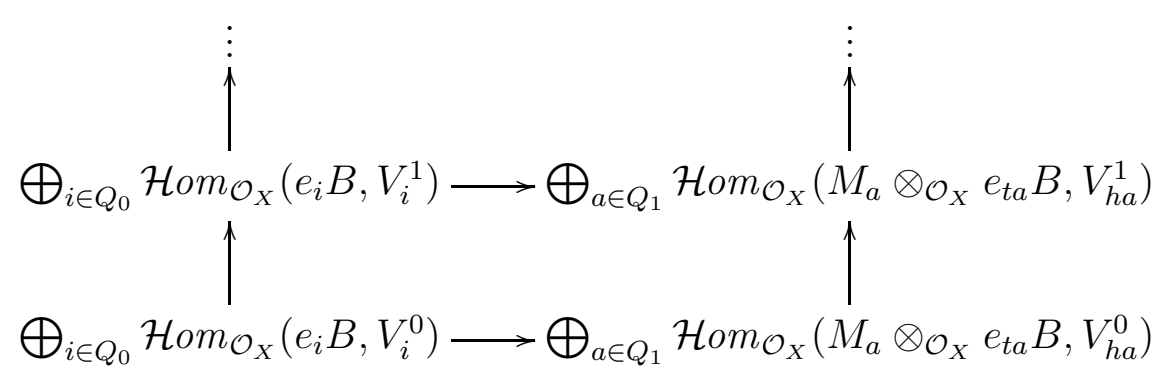

The horizontal differentials are $(-1)^{q} d$, where $d$ is the map 3.3 for $V^{q}$, and the vertical differentials are induced by $\delta: V_{i}^{n} \longrightarrow V_{i}^{n+1}$. Using the fact that the $\delta$ are $B$-module homomorphisms it is easy to check that the squares of the double complex anti-commute.

Denote the total complex of $C(V)^{\bullet \bullet}$ by $I(V)^{\bullet}$ and note that we have a canonical injection of $B$-modules

$$
\bar{\epsilon}: V \longrightarrow I(V)^{0}=\bigoplus_{i \in Q_{0}} \mathcal{H o m}_{\mathcal{O}_{X}}\left(e_{i} B, V_{i}^{0}\right)
$$


where $\bar{\epsilon}$ is given by composing

$$
\epsilon: V^{0} \longrightarrow \bigoplus_{i \in Q_{0}} \mathcal{H}{ }^{0} m_{\mathcal{O}_{X}}\left(e_{i} B, V_{i}^{0}\right)
$$

defined in (3.2) with the inclusion $V \hookrightarrow V^{0}$.

Proposition 3.5. For any B-module $V$, the complex $I(V)^{\bullet}$ is an injective resolution of $V$.

Proof. It follows from Proposition 3.4 that each term in (3.5) is injective and so $I(V)^{n}$ is an injective $B$-module for each $n$. We have just seen that $V$ injects into $I(V)^{0}$, so all that remains to check is that $V \rightarrow I(V)^{\bullet}$ is an exact complex. We use the following notation:

$$
\begin{aligned}
F(V) & =\bigoplus_{i \in Q_{0}} \mathcal{H o m}_{\mathcal{O}_{X}}\left(e_{i} B, V_{i}\right) \\
G(V) & =\bigoplus_{a \in Q_{1}} \mathcal{H} m_{\mathcal{O}_{X}}\left(M_{a} \otimes_{\mathcal{O}_{X}} e_{t a} B, V_{h a}\right)
\end{aligned}
$$

Then we have the following commutative diagram:

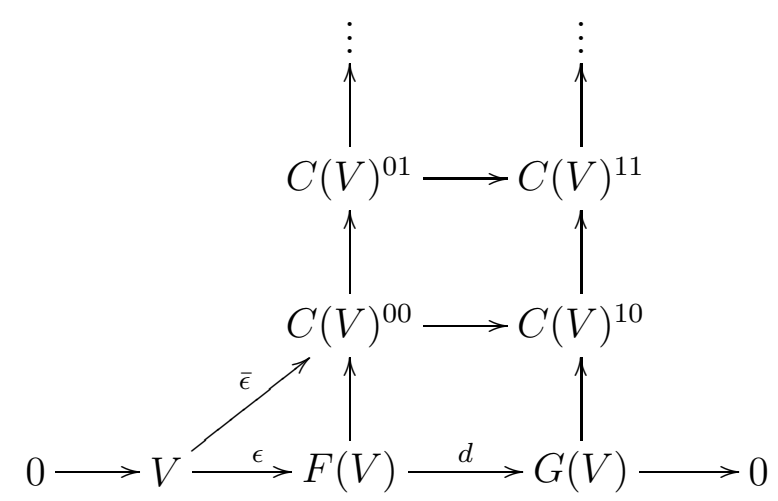

Since, for any $i \in Q_{0}$ and $a \in Q_{1}$, the $\mathcal{O}_{X}$-modules $e_{i} B$ and $M_{a} \otimes_{\mathcal{O}_{X}} e_{t a} B$ are locally free, we see that $\mathcal{H o m}_{\mathcal{O}_{X}}\left(e_{i} B,-\right)$ and $\mathcal{H o m}_{\mathcal{O}_{X}}\left(M_{a} \otimes_{\mathcal{O}_{X}} e_{i} B,-\right)$ are exact functors. Hence the columns of the above diagram are exact. Furthermore, the bottom row of the diagram is exact from Proposition [3.1. From these two facts it is easy to see that $V \rightarrow I(V)^{\bullet}$ is an exact complex.

\section{A long exact sequence}

Now that we can take injective resolutions of $B$-modules, we may define $\operatorname{Ext}_{B}^{i}(V, W)$ as the $i$ th cohomology of the complex $\operatorname{Hom}_{B}\left(V, I(W)^{\bullet}\right)$, where 
$I(W)^{\bullet}$ is an injective resolution of $W$. Our main theorem provides a tool for calculating $\operatorname{Ext}_{B}^{i}(V, W)$, in terms of the Ext groups of the $\mathcal{O}_{X}$-modules $V_{i}$ and $W_{j}$, via a long exact sequence. As explained in the Introduction, several earlier results are special cases of this.

Before stating the result, we need to explain how to interpret some of the notation. We are already familiar with the map

$$
\operatorname{Hom}(E, F) \rightarrow \operatorname{Hom}\left(M_{a} \otimes E, M_{a} \otimes F\right): f \mapsto 1 \otimes f
$$

given by the functor $E \mapsto M_{a} \otimes E$. Since $M_{a}$ is locally free, this functor is exact and hence it also induces a map

$$
\operatorname{Ext}^{i}(E, F) \rightarrow \operatorname{Ext}^{i}\left(M_{a} \otimes E, M_{a} \otimes F\right)
$$

which we shall also denote by $f \mapsto 1 \otimes f$. This map is easily understood in the Yoneda picture, where Ext $^{i}$ classifies exact sequences from $F$ to $E$ with $i$ intermediate terms. However, for our purposes we need the following equivalent formulation. If $F^{\bullet}$ is any resolution of $F$ and $\widetilde{f} \in \operatorname{Hom}\left(E, F^{i}\right)$ is a cocyle representing $f \in \operatorname{Ext}^{i}(E, F)$, then $1 \otimes \widetilde{f} \in \operatorname{Hom}\left(M_{a} \otimes E, M_{a} \otimes F^{i}\right)$ is a cocyle representing $1 \otimes f \in \operatorname{Ext}^{i}\left(M_{a} \otimes E, M_{a} \otimes F\right)$.

With this understood, we can state and prove our theorem. The context is the general one of Section [3] $\left(X, \mathcal{O}_{X}\right)$ is a ringed space, $Q$ is a quiver and $M_{a}$ are locally free $\mathcal{O}_{X}$-module for each $a \in Q_{1}$. Then $B$ is the $M$-twisted path algebra over $X$.

Theorem 4.1. For any $B$-modules $V$ and $W$, there is a long exact sequence

$$
\begin{aligned}
0 & \rightarrow \operatorname{Hom}_{B}(V, W) \rightarrow \bigoplus_{i \in Q_{0}} \operatorname{Hom}_{\mathcal{O}_{X}}\left(V_{i}, W_{i}\right) \stackrel{\partial}{\longrightarrow} \bigoplus_{a \in Q_{1}} \operatorname{Hom}_{\mathcal{O}_{X}}\left(M_{a} \otimes V_{t a}, W_{h a}\right) \\
& \rightarrow \operatorname{Ext}_{B}^{1}(V, W) \rightarrow \bigoplus_{i \in Q_{0}} \operatorname{Ext}_{\mathcal{O}_{X}}^{1}\left(V_{i}, W_{i}\right) \stackrel{\partial}{\longrightarrow} \bigoplus_{a \in Q_{1}} \operatorname{Ext}_{\mathcal{O}_{X}}^{1}\left(M_{a} \otimes V_{t a}, W_{h a}\right) \\
& \rightarrow \operatorname{Ext}_{B}^{2}(V, W) \rightarrow \cdots
\end{aligned}
$$

The maps $\partial$ are given by

$$
\partial:\left(f_{i}\right)_{i \in Q_{0}} \longmapsto\left(f_{h a} \circ \phi_{a}-\psi_{a} \circ\left(1 \otimes f_{t a}\right)\right)_{a \in Q_{1}}
$$

where o is the Yoneda product.

Proof. Let $I(W)^{\bullet}$ be the injective resolution of $W$ given by Proposition 3.5, i.e. the total complex of the double complex $C(W)^{\bullet \bullet}$ of (3.5). Then the group $\operatorname{Ext}_{B}^{i}(V, W)$ is the $i$ th cohomology group of the complex $\operatorname{Hom}_{B}\left(V, I(W)^{\bullet}\right)$, 
which can be computed from the spectral sequence for the double complex $\operatorname{Hom}_{B}\left(V, C(W)^{\bullet \bullet}\right)$. Using the adjunction of Corollary 3.3, this double complex can be written

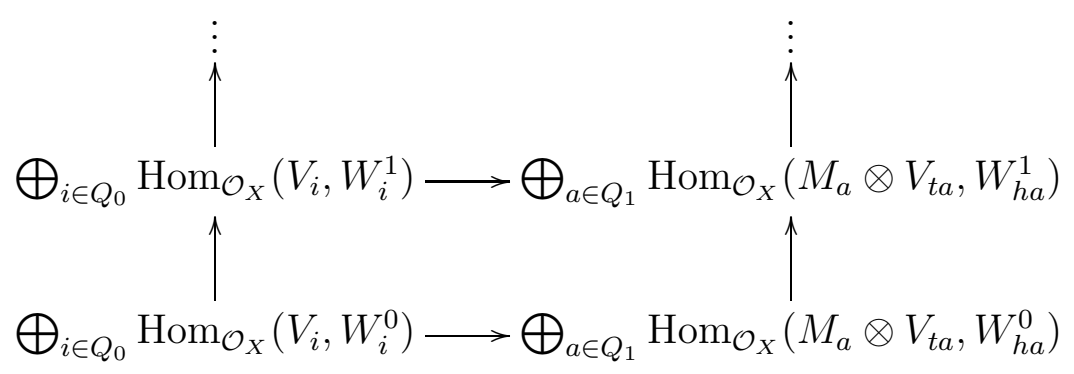

If we pass to the $E_{1}$ term of the spectral sequence by computing vertical cohomology, then we obtain the required groups

$$
\bigoplus_{i \in Q_{0}} \operatorname{Ext}_{\mathcal{O}_{X}}^{q}\left(V_{i}, W_{i}\right) \text { and } \bigoplus_{a \in Q_{1}} \operatorname{Ext}_{\mathcal{O}_{X}}^{q}\left(M_{a} \otimes V_{t a}, W_{h a}\right)
$$

The fact that we started with a two column complex means that the spectral sequence converges at the $E_{2}$ term and yields precisely the long exact sequence of the theorem.

To see that the maps are as claimed, observe that the horizontal maps in (4.1) are given by

$$
\left(\widetilde{f}_{i}\right)_{i \in Q_{0}} \longmapsto\left(\widetilde{f}_{h a} \circ \phi_{a}-\psi_{a}^{q} \circ\left(1 \otimes \tilde{f}_{t a}\right)\right)_{a \in Q_{1}}
$$

where $\tilde{f}_{i} \in \operatorname{Hom}\left(V_{i}, W_{i}^{q}\right)$ is a cocycle representing $f_{i} \in \operatorname{Ext}^{q}\left(V_{i}, W_{i}\right)$, while $\psi_{a}^{q} \in \operatorname{Hom}\left(M_{a} \otimes W_{t a}^{q}, W_{h a}^{q}\right)$ are the lifts of $\psi_{a} \in \operatorname{Hom}\left(M_{a} \otimes W_{t a}, W_{h a}\right)$, as in (3.4). In (4.2) the symbol $\circ$ just denotes composition of maps.

The first composite $\widetilde{f}_{h a} \circ \phi_{a}$ is immediately seen to be a cocyle representing the Yoneda product $f_{h a} \circ \phi_{a}$. For the second composite, observe, from the remark preceding the statement of the theorem, that

$$
1 \otimes \widetilde{f}_{t a} \in \operatorname{Hom}\left(M_{a} \otimes V_{t a}, M_{a} \otimes W_{t a}^{q}\right)
$$

is a cocyle representing $1 \otimes f_{t a} \in \operatorname{Ext}^{q}\left(M_{a} \otimes V_{t a}, M_{a} \otimes W_{t a}\right)$ and thus that the composite $\psi_{a}^{q} \circ\left(1 \otimes \widetilde{f}_{t a}\right)$ is a cocyle representing the Yoneda product $\psi_{a} \circ\left(1 \otimes f_{t a}\right)$

Note for the careful reader: because it makes no difference to the result, for simplicity we have not carried the signs $(-1)^{q}$ attached to the maps $d$ of (3.5) through to the maps $\partial$ of the theorem. 


\section{Hypercohomology}

Let $V$ and $W$ be $B$-modules. When $V$ is locally free, we show in this section how to give a description of $\operatorname{Ext}^{i}(V, W)$ in terms of hypercohomology.

Consider the exact sequence of sheaves given by Proposition 3.1

$$
\begin{aligned}
0 \longrightarrow W \stackrel{\epsilon}{\longrightarrow} \bigoplus_{i \in Q_{0}} \mathcal{H o m}_{\mathcal{O}_{X}}\left(e_{i} B, W_{i}\right) \\
\stackrel{d}{\longrightarrow} \bigoplus_{a \in Q_{1}} \mathcal{H o m}_{\mathcal{O}_{X}}\left(M_{a} \otimes_{\mathcal{O}_{X}} e_{t a} B, W_{h a}\right) \longrightarrow 0
\end{aligned}
$$

Applying the functor $\mathcal{H} \mathrm{mm}_{B}(V,-)$ and using Corollary 3.3 we obtain the exact sequence

$$
\begin{aligned}
0 \longrightarrow \mathcal{H o m}_{B}(V, W) \longrightarrow \bigoplus_{i \in Q_{0}} \mathcal{H}^{0} m_{\mathcal{O}_{X}}\left(V_{i}, W_{i}\right) \\
\stackrel{\delta}{\longrightarrow} \bigoplus_{a \in Q_{1}} \mathcal{H} \operatorname{mom}_{\mathcal{O}_{X}}\left(M_{a} \otimes_{\mathcal{O}_{X}} V_{t a}, W_{h a}\right),
\end{aligned}
$$

where $\delta$ is given by

$$
\left(f_{i}\right)_{i \in Q_{0}} \longmapsto\left(f_{h a} \circ \phi_{a}-\psi_{a} \circ\left(1 \otimes f_{t a}\right)\right)_{a \in Q_{1}}
$$

The complex of sheaves

$$
C^{\bullet}(V, W): \quad \bigoplus_{i \in Q_{0}} \mathcal{H}^{\circ} m_{\mathcal{O}_{X}}\left(V_{i}, W_{i}\right) \stackrel{\delta}{\longrightarrow} \bigoplus_{a \in Q_{1}} \mathcal{H} m_{\mathcal{O}_{X}}\left(M_{a} \otimes_{\mathcal{O}_{X}} V_{t a}, W_{h a}\right)
$$

formed by the last two terms of this sequence is important because of the following result.

Theorem 5.1. Let $\left(X, \mathcal{O}_{X}\right)$ be a ringed space, let $Q$ be a quiver, let $M_{a}$ be a locally free $\mathcal{O}_{X}$-module for each $a \in Q_{1}$ and let $B$ be the $M$-twisted path algebra over $X$. Let $V$ and $W$ be $B$-modules and suppose that $V$ is locally free. Then

$$
\operatorname{Ext}_{B}^{p}(V, W) \cong \mathbb{H}^{p}\left(C^{\bullet}(V, W)\right) .
$$

We shall need the following three lemmas for the proof.

Lemma 5.2. Let $V$ and $W$ be B-modules and consider the two term complex obtained by applying the global sections functor $\Gamma$ to $C^{\bullet}(V, W)$. Then

$$
H^{0}\left(\Gamma\left(C^{\bullet}(V, W)\right)\right) \cong \operatorname{Hom}_{B}(V, W) .
$$


If, moreover, $W$ is injective, then

$$
H^{p}\left(\Gamma\left(C^{\bullet}(V, W)\right)\right)=0
$$

for $p \geqslant 1$.

Proof. Observe that the complex $\Gamma\left(C^{\bullet}(V, W)\right)$ coincides with the part of the long exact sequence of Theorem 4.1 made up by the second and third term. The result follows.

Lemma 5.3. Let $W$ be an injective B-module. Then each $W_{i}$ is an injective $\mathcal{O}_{X}$-module.

Proof. We claim that the left adjoint of the forgetful functor $W \mapsto W_{i}$ from $B$-modules to $\mathcal{O}_{X}$-modules is the functor $L \mapsto B e_{i} \otimes L$. This latter functor is exact because the $M_{a}$ are locally free and hence its right adjoint (the forgetful functor) preserves injectives. Thus the lemma follows from the claim.

In order to prove the claim we use the adjunction

$$
\operatorname{Hom}_{B}(V \otimes L, W) \cong \operatorname{Hom}_{\mathcal{O}_{X}}\left(L, \operatorname{Hom}_{B}(V, W)\right)
$$

for an $\mathcal{O}_{X}$-module $L$ and $B$-modules $V$ and $W$ : taking $V=B e_{i}$ it follows that the functor $L \mapsto B e_{i} \otimes L$ is the left adjoint of $W \mapsto \operatorname{Hom}_{B}\left(B e_{i}, W\right)$. We finish the proof by observing that there is a natural isomorphism $W_{i}=$ $e_{i} W \stackrel{\cong}{\rightarrow} \operatorname{Hom}_{B}\left(B e_{i}, W\right)$ given by $e_{i} w \mapsto\left(x e_{i} \mapsto x w\right)$, with inverse $\alpha \mapsto$ $\alpha\left(e_{i}\right)=e_{i}\left(\alpha\left(e_{i}\right)\right)$.

Lemma 5.4. Let $L$ and $M$ be locally free $\mathcal{O}_{X}$-modules and let $I$ be an injective $\mathcal{O}_{X}$-module. Then $\mathcal{H o m}_{\mathcal{O}_{X}}(M \otimes L, I)$ is an injective $\mathcal{O}_{X}$-module.

Proof. This follows from the standard adjunction

$$
\operatorname{Hom}_{\mathcal{O}_{X}}\left(N, \mathcal{H o m}_{\mathcal{O}_{X}}(M \otimes L, I)\right) \cong \operatorname{Hom}_{\mathcal{O}_{X}}(M \otimes L \otimes N, I)
$$

by an argument similar to that used in the proof of Propositions 2.5 and 3.4.

Proof of Theorem 5.1. We begin by noting that the statement is true for $p=0$. For any complex of sheaves $\mathcal{F}^{\bullet}$ one has $\mathbb{H}^{0}\left(\mathcal{F}^{\bullet}\right)=H^{0}\left(\Gamma\left(\mathcal{F}^{\bullet}\right)\right)$ and therefore we have, using Lemma 5.2, that

$$
\mathbb{H}^{0}\left(C^{\bullet}(V, W)\right) \cong H^{0}\left(\Gamma\left(C^{\bullet}(V, W)\right)\right) \cong \operatorname{Hom}_{B}(V, W) .
$$

Thus it suffices to show that the functor $\mathbb{H}^{p}\left(C^{\bullet}(V,-)\right)$ on $B$-modules is a universal $\delta$-functor. Let $0 \rightarrow W^{\prime} \rightarrow W \rightarrow W^{\prime \prime} \rightarrow 0$ be a short exact 
sequence of $B$-modules. Then, because $M_{a}$ and $V$ are locally free, we get a corresponding short exact sequence of complexes

$$
0 \longrightarrow C^{\bullet}\left(V, W^{\prime}\right) \longrightarrow C^{\bullet}(V, W) \longrightarrow C^{\bullet}\left(V, W^{\prime \prime}\right) \longrightarrow 0
$$

Hence, by general properties of hypercohomology, we get the required long exact sequence

$$
\begin{aligned}
0 & \longrightarrow \mathbb{H}^{0}\left(C^{\bullet}\left(V, W^{\prime}\right)\right) \longrightarrow \mathbb{H}^{0}\left(C^{\bullet}(V, W)\right) \longrightarrow \mathbb{H}^{0}\left(C^{\bullet}\left(V, W^{\prime \prime}\right)\right) \\
& \longrightarrow \mathbb{H}^{1}\left(C^{\bullet}\left(V, W^{\prime}\right)\right) \longrightarrow \cdots
\end{aligned}
$$

natural with respect to morphisms of short exact sequences. Thus $\mathbb{H}^{p}\left(C^{\bullet}(V,-)\right)$ is a $\delta$-functor.

It remains to show that $\mathbb{H}^{p}\left(C^{\bullet}(V,-)\right)$ is universal. Since the category of $B$-modules has enough injectives it suffices to show that for any injective $B$-module $I$, one has $\mathbb{H}^{p}\left(C^{\bullet}(V, I)\right)=0$ for all $p \geqslant 1$. Consider the first hypercohomology spectral sequence

$$
{ }^{I} E_{2}^{p q}=H^{p}\left(R^{q} \Gamma\left(C^{\bullet}(V, I)\right)\right) \Longrightarrow \mathbb{H}^{p+q} C^{\bullet}(V, I) .
$$

(Here we have denoted sheaf cohomology by $R^{q} \Gamma$ to avoid confusion, i.e., $R^{q} \Gamma\left(C^{\bullet}(V, I)\right)$ is the complex obtained from $C^{\bullet}(V, I)$ by taking the $q$ th sheaf cohomology group of each term.) By Lemma 5.3 we have that each $I_{i}$ is an injective $\mathcal{O}_{X}$-module. Hence Lemma [5.4 shows that each term of $C^{\bullet}(V, I)$ is an injective $\mathcal{O}_{X}$-module and thus has vanishing higher sheaf cohomology. It follows that the spectral sequence collapses at the $E_{2}$-term and we get

$$
\mathbb{H}^{p}\left(C^{\bullet}(V, I)\right)=H^{p}\left(\Gamma\left(C^{\bullet}(V, I)\right)\right)
$$

which vanishes for $p \geqslant 1$ by Lemma 5.2. This finishes the proof.

\section{References}

[1] L. Álvarez-Cónsul and O. García-Prada, Dimensional reduction, $\mathrm{SL}(2, \mathbb{C})$-equivariant bundles and stable holomorphic chains, Int. J. Math. 12 (2001), 159-201.

[2] L. Álvarez-Cónsul and O. García-Prada, Dimensional reduction and quiver bundles, J. Reine Angew. Math. 556 (2003), 1-46.

[3] L. Álvarez-Cónsul and O. García-Prada, Hitchin-Kobayashi correspondence, quivers and vortices, Comm. Math. Phys. 238 (2003), 1-33. 
[4] M. Auslander, I. Reiten, and S. O. Smalø, Representation theory of Artin algebras, Cambridge Studies in Advanced Mathematics, vol. 36, Cambridge University Press, 1995.

[5] D. J. Benson, Representations and cohomology I, 2nd ed., Cambridge Studies in Advanced Mathematics, vol. 30, Cambridge University Press, 1998.

[6] I. Biswas and S. Ramanan, An infinitesimal study of the moduli of Hitchin pairs, J. London Math. Soc. (2) 49 (1994), 219-231.

[7] F. Bottacin, Symplectic geometry on moduli spaces of stable pairs, Ann. Sci. Ec. Norm. Super., IV. Ser. 28 (1995), 391-433.

[8] S. B. Bradlow, Special metrics and stability for holomorphic bundles with global sections, J. Differential Geom. 33 (1991), 169-213.

[9] S. B. Bradlow and O. García-Prada, Stable triples, equivariant bundles and dimensional reduction, Math. Ann. 304 (1996), 225-252.

[10] S. B. Bradlow, O. García-Prada, and P. B. Gothen, Moduli spaces of holomorphic triples over compact Riemann surfaces, Math. Ann. 328 (2004), 299-351.

[11] S. B. Bradlow, O. García-Prada, and P. B. Gothen, Surface group representations in $\mathrm{PU}(p, q)$ and Higgs bundles, J. Diff. Geom. 64 (2003), $111-170$.

[12] W. Crawley-Boevey, Lectures on representations of quivers, URL: http://www.amsta.leeds.ac.uk/ pmtwc/quivlecs.ps, 1992.

[13] P. Gabriel and A. V. Roiter, Representations of finite-dimensional algebras, Encyclopaedia of Mathematical Sciences, vol. 73, pp. 1-177, Springer, 1992.

[14] O. García-Prada, Dimensional reduction of stable bundles, vortices and stable pairs, Int. J. Math. 5 (1994), 1-52.

[15] S. I. Gelfand and Y. I. Manin, Methods of homological algebra, Grundlehren der Mathematischen Wissenschaften, vol. 233, Springer, 1996.

[16] R. Hartshorne, Algebraic geometry, Graduate Texts in Mathematics, vol. 52, Springer, 1977. 
[17] T. Hausel and M. Thaddeus, Generators for the cohomology ring of the moduli space of rank 2 Higgs bundles, Proc. London Math. Soc. (3) 88 (2004) 632-658.

[18] N. J. Hitchin, The self-duality equations on a Riemann surface, Proc. London Math. Soc. (3) 55 (1987), 59-126.

[19] E. Markman, Spectral curves and integrable systems, Compositio Math. 93 (1994), 255-290.

[20] N. Nitsure, Moduli of semistable pairs on a curve, Proc. London Math. Soc. (3) 62 (1991), 275-300.

[21] J. P. Serre, Faisceaux algébriques cohérents, Ann. Math. 61 (1955), $197-$ 278.

[22] M. Thaddeus, Stable pairs, linear systems and the Verlinde formula, Invent. Math. 117 (1994), 317-353.

Departamento de Matemática Pura

Faculdade de Ciências

Universidade do Porto

Rua do Campo Alegre

4169-007 Porto, Portugal

E-mail: pbgothen@fc.up.pt
Mathematical Sciences

University of Bath

Claverton Down

Bath BA2 7AY, U.K.

E-mail: A.D.King@maths.bath.ac.uk 